\title{
Qualidade de vida de pacientes com esquizofrenia internados em hospital de custódia*
}

\author{
An evaluation of the quality of life of schizophrenic \\ patients admitted to a forensic hospital \\ Ana Flávia Ferreira de Almeida Santana', Tânia Couto Machado Chianca², Clareci Silva Cardoso³
}

\section{RESUMO}

Objetivo: Investigar a qualidade de vida (QV) de pacientes com o diagnóstico de esquizofrenia que cumprem medida de segurança em regime fechado. Métodos: Estudo epidemiológico com delineamento transversal, realizado com pacientes de um hospital de custódia. As entrevistas foram conduzidas utilizando questionário sociodemográfico e a escala de qualidade de vida Quality of Life Scale (QLS-BR), específica para pacientes com diagnóstico de esquizofrenia. Foi realizada análise descritiva e multivariada. A associação entre os escores de QV e as características clínicas, sociodemográficas e do delito foi obtida por análise multivariada, através da árvore de decisão, por meio do algoritmo Chi-squared Automatic Interaction Detector (CHAID). Resultados: Participaram deste estudo 54 pacientes, sendo $90,7 \%$ do sexo masculino, com a média de idade de 40 anos. Todos os domínios de qualidade de vida, inclusive o global, apresentaram escores compatíveis com uma QV muito baixa. O domínio ocupacional apresentou-se como o mais comprometido. Maior duração da doença, ter cometido homicídio e estar com idade superior a 40 anos foram as

\section{Palavras-chave}

Esquizofrenia, qualidade de vida, medida de segurança, enfermagem. variáveis associadas a uma baixa qualidade de vida pela análise multivariada. Conclusão: Evidenciou-se uma baixa QV dos pacientes que cumprem medida de segurança em regime fechado, realidade que precisa ser modificada a partir da viabilização de mudanças nas políticas brasileiras.

\section{ABSTRACT}

Objective: To investigate the quality of life (QOL) in patients diagnosed with schizophrenia that are under involuntary commitment. Methods: A cross-sectional epidemiological study was carried out on patients from a penitentiary hospital. The survey was conductid by a social-demographic questionnaire and the Quality of Life Scale (QLS-BR), specific for patients diagnosed with schizophrenia. A descriptive and multivariate analysis was performed. The association between the scores of QOL and the clinical, social-demographic and felony characteristics was obtained by multivariate analysis, using a resource the decision tree, by means of the Chi-squared Automatic Interaction Detector (CHAID) algorithm. Results: 54 people participated in this study, of which $90.7 \%$ were male, with an average age of 40 years old. All the domains of quality of life, inclu- 


\section{Keywords}

Schizophrenia, quality of life, custody order, nursing. ding the global one, presented scores compatible with a very low QOL. The occupational domain showed to be the most affected one. Long lasting disease, having commited homicide and being over 40 years old were the variables associated to a low quality of life in the multivariate analysis.

Conclusion: A low QOL was noticed among the patients that are under involuntary commitment, a reality that needs to be modified by making possible changes in Brazilian policies.

\section{INTRODUÇÃO}

Do ponto de vista jurídico, o portador de doença mental ou comprometimento cognitivo, comprovado por perícia médica, ao cometer um crime, não é considerado autor do ato, por ser julgado inimputável, incapaz de distinguir o caráter ilícito dos próprios atos. Nesses casos, a lei determina a absolvição com aplicação de medida de segurança (MS), com prazo indeterminado, estando sujeito à perícia médica indicatória de cessação de periculosidade!

A aplicação da MS foi destinada ao inimputável, aquele que em função do seu comprometimento não pode ser considerado culpado, mas representa perigo para a sociedade. A profilaxia social, direcionada aos cuidados dos portadores de transtornos psiquiátricos, permitiu que a MS, no campo do direito penal, funcionasse como um dispositivo de atuação sobre esses doentes. Os manicômios judiciais no Brasil passaram, então, a ser projetados para aqueles que comprometiam o funcionamento e as intenções da defesa social ${ }^{2}$.

Os manicômios brasileiros são um híbrido social (parte residência e parte organização formal) que mantém uma tensão entre o mundo doméstico e o institucional. Diante das circunstâncias, o manicômio judiciário tem assumido características tanto de presídios como de asilos. Portanto, essas instituições mostram uma dupla vertente caracterizada pela sobreposição de um espaço prisional a um asilar, penitenciário a hospitalar ${ }^{3}$.

A esquizofrenia é a doença paradigmática da psiquiatria. É uma síndrome clínica complexa, considerada na literatura como o mais grave dos transtornos psiquiátricos. De caráter crônico, pode gerar sérios prejuízos na vida dos pacientes e dos familiares. O impacto da doença provoca perdas funcionais, relacionadas a habilidades de trabalho, afetivas e de relacionamento social. Perdas objetivas e subjetivas são percebidas pelos próprios pacientes como fatores de interferência na qualidade de vida (QV) ${ }^{4,56}$.

Nesse contexto, é pertinente a realização da avaliação da QV dos pacientes que estão sob sanção penal de MS, uma vez que os mesmos têm recebido tanto tratamentos ambulatoriais como em regime fechado no Brasil. Entendese QV como a percepção do indivíduo acerca de sua posição na vida, levando em consideração o contexto cultural, sistema de valores construído e vivenciado em relação a objetivos, expectativas, padrões e preocupações ${ }^{7-9}$. Tal avaliação é de grande importância para o processo de construção e aprimoramento dos programas e das instituições que oferecem assistência aos pacientes portadores de transtornos psiquiátricos. Estudos de QV dos pacientes judiciários podem apontar indicadores que subsidiem a avaliação e planejamento das ações envolvidas no âmbito jurídico.

Diante disso, a proposta deste estudo é investigar a QV de pacientes judiciários com diagnóstico de esquizofrenia que cumprem MS em regime fechado em uma instituição brasileira.

\section{MÉTODOS}

Foi realizado um estudo epidemiológico com delineamento transversal em hospital de custódia de Minas Gerais, Brasil. Essa instituição, assim como outras no Brasil, tem como objetivo fazer cumprir ordem judicial de medida de segurança.

Os pacientes foram selecionados de acordo com os seguintes critérios de elegibilidade: diagnóstico de esquizofrenia pelo Código Internacional de Doenças (CID); idades entre 18 e 55 anos; mínimo de 3 meses de institucionalização; quadro estável da patologia durante as 3 semanas que antecederam a entrevista; ausência de transtorno neurológico ou retardo mental; ausência de histórico de dependência de substância química nos últimos 6 meses, 70,11 .

A seleção dos pacientes foi baseada em pesquisa nos processos onde se encontravam os laudos médicos e nos prontuários do hospital. O diagnóstico, assim como a ausência de transtorno neurológico, retardo mental e dependência de substância química ilícita foram avaliados em laudos médicos oficiais e nos prontuários de acompanhamento. O diagnóstico de esquizofrenia foi baseado na última avaliação realizada por perito judicial, nos últimos 6 meses, e confirmado em prontuário no hospital de custódia. A estabilidade da patologia foi avaliada por meio de informações obtidas com os profissionais de saúde e agentes penitenciários responsáveis pelo paciente.

Os pacientes foram convidados a participar do estudo, pessoalmente, por um membro da equipe. Nos casos em 
que o convite foi aceito, os pacientes foram informados sobre os objetivos e a importância do estudo.

A pesquisa foi conduzida de acordo com as diretrizes e normas da Resolução no 196/1996, do Conselho Nacional de Saúde Brasileiro e da Resolução no 251, de 7/08/1997, do mesmo conselho, para atender às exigências da Declaração de Helsink. Este estudo foi aprovado pelo Comitê de Ética e Pesquisa da Universidade Federal de Minas Gerais (COEP/ UFMG), Parecer no 389/2007, com termo de consentimento livre e esclarecido (TCLE) para o paciente e curador.

As entrevistas individuais foram realizadas por uma psicóloga previamente treinada. Foram aplicados dois instrumentos: um questionário sociodemográfico e a escala de qualidade de vida Quality of Life Scale - Brasil (QLS-BR) ${ }^{10}$.

Foram levantadas informações acerca do diagnóstico dos pacientes, tais como: história clínica, características pessoais e demográficas, duração da doença, tempo de internação em hospitais psiquiátricos; e, também, informações sobre a situação jurídica dos pacientes.

A escala QLS-BR foi utilizada para mensurar a QV dos pacientes. Trata-se de um instrumento específico para pacientes com diagnóstico de esquizofrenia. Foi traduzida e validada para o Brasil e apresentou boas qualidades psicométricas ${ }^{710}$. Durante o processo de validação desse instrumento para o Brasil ${ }^{7,10}$, bem como do seu uso em outras investigações ${ }^{12}$, não foram encontrados problemas em relação à compreensão das questões por parte dos pacientes, mesmo em casos de baixa escolaridade ou analfabetismo.

A escala é composta por 21 itens, distribuídos em 3 domínios: social, ocupacional e intrapsíquico e de relações interpessoais. Os itens abrangem informações sobre sintomatologia e funcionamento do paciente nas 3 semanas precedentes à entrevista. A grade de cotação para cada item é formada por um escalonamento tipo Likert de 7 pontos. Os escores 5 e 6 apontam um funcionamento normal ou inalterado, os escores de 2 a 4 refletem prejuízo em determinado aspecto da vida do paciente e os escores 0 e 1 revelam prejuízo considerável do item investigado.

O programa Epi-data foi utilizado para entrada de dados. Todos os questionários foram inseridos na planilha por dupla digitação. As análises estatísticas foram realizadas no Statistic Package for Social Sciences (SPSS) versão 11.0.

Utilizou-se a estatística descritiva, com distribuição de frequências, medidas de tendência central e dispersão. Quando necessário, as variáveis ordinais foram categorizadas tendo como referência o valor da mediana.

Quando pertinente, os escores de QV na QLS-BR foram codificados em 3 categorias: QV muito comprometida, QV comprometida e QV inalterada.

Para avaliar a associação entre a QV e as características sociodemográficas, clínicas e do delito foi empregada análise multivariada, utilizando a árvore de decisão por meio do algoritmo Chi-squared Automatic Interaction Detector (CHAID), por meio de um esquema descritivo. Esse método consiste em sucessivas divisões no conjunto de dados, de forma a torná-lo cada vez mais homogêneo em relação à variável resposta ${ }^{13}$. A árvore de decisão utiliza-se de um gráfico que ilustra regras de decisão que começa com um nó raiz, onde todas as observações da amostra são apresentadas. Os nós produzidos em sequência representam subdivisões dos dados em grupos cada vez mais homogêneos. A árvore de decisão seleciona um subconjunto de preditores dentre um grande conjunto de variáveis que é usado na construção de um modelo paramétrico formal. O método ainda identifica relacionamentos que pertencem a apenas um subgrupo específico e recodifica variáveis categóricas e contínuas com o mínimo de perda de informação ${ }^{13}$.

Nas análises, a variável resposta foi definida como o escore global de qualidade de vida dos pacientes na forma dimensional, sem categorizações. As variáveis explicativas consideradas foram de natureza sociodemográfica, clínica e relacionadas à internação e cumprimento da medida de segurança, e foram tratadas de forma dicotômica ${ }^{13}$.

Para o CHAID, todas as variáveis entraram inicialmente na árvore de decisão. $O$ valor $-p \leq 0,05$ da estatística quiquadrado usando a correção de Bonferroni foi o principal critério de parada adotado. O ajuste do modelo final foi avaliado por meio da estatística de risco que compara a diferença entre o valor esperado e o observado pelo modelo, indicando em que medida a árvore corretamente prediz os resultados ${ }^{13}$.

\section{RESULTADOS}

No momento da coleta de dados, 178 pacientes estavam hospitalizados em cumprimento de MS, sendo 63 deles elegíveis para o estudo. Um total de 6 pacientes não aceitou participar, 2 estavam internados em outra instituição para tratamento de problemas clínicos e a equipe sugeriu a exclusão de um paciente em função de quadro de agressividade. Dentre aqueles que preenchiam os critérios de elegibilidade, houve uma perda de 14\%. Foi feita comparação das perdas em relação às variáveis sexo, idade, diagnóstico, tempo de hospitalização e tipo de delito. As análises não apontaram perda diferenciada ( $p>0,05$ ).

Foram entrevistados 54 pacientes e a entrevista teve duração média de 45 minutos. A maioria dos pacientes eram homens (90,7\%), com a média de idade de 40 anos. As Tabelas 1 e 2 trazem informações sociodemográficas e clínicas da população estudada. Em relação à escolaridade, 69,2\% dos pacientes não concluíram o ensino fundamental e $20 \%$ são analfabetos. Em relação ao estado conjugal, 83,3\% dos pacientes relataram não possuir parceiro (Tabela 1). 
Tabela 1. Distribuição dos pacientes nas variáveis sociodemográficas $(n=54)$

\begin{tabular}{|c|c|c|}
\hline Variáveis sociodemográficas & $\mathrm{n}$ & $\%$ \\
\hline \multicolumn{3}{|l|}{ Sexo } \\
\hline Masculino & 49 & 90,7 \\
\hline Feminino & 5 & 9,3 \\
\hline \multicolumn{3}{|l|}{ Idade } \\
\hline$<40$ anos & 23 & 42,6 \\
\hline$\geq 40$ anos & 31 & 57,4 \\
\hline \multicolumn{3}{|l|}{ Escolaridade } \\
\hline Analfabeto & 11 & 20,4 \\
\hline Ensino fundamental incompleto & 36 & 66,7 \\
\hline Ensino fundamental completo ou + & 5 & 9,3 \\
\hline \multicolumn{3}{|l|}{ Situação Conjugal } \\
\hline Sem parceiro & 45 & 83,3 \\
\hline Com parceiro & 9 & 16,7 \\
\hline \multicolumn{3}{|l|}{ Renda Familiar } \\
\hline$\leq 2$ salários & 9 & 16,7 \\
\hline$>2$ salários & 5 & 9,3 \\
\hline Não soube informar & 40 & 74,1 \\
\hline \multicolumn{3}{|l|}{ Aposentadoria } \\
\hline Sim & 4 & 7,4 \\
\hline Não & 50 & 92,6 \\
\hline \multicolumn{3}{|l|}{ Ajuda Financeira } \\
\hline Sim & 12 & 22,2 \\
\hline Não & 42 & 77,8 \\
\hline \multicolumn{3}{|l|}{ Atividade profissional* } \\
\hline Níveis 1 e 2 competência & 52 & 96,3 \\
\hline Demais níveis competência & 2 & 3,7 \\
\hline \multicolumn{3}{|l|}{ Tempo que parou de trabalhar } \\
\hline$<12$ anos & 25 & 46,3 \\
\hline$\geq 12$ anos & 28 & 51,9 \\
\hline
\end{tabular}

*A atividade profissional tem como referência a Classificação Internacional Uniforme de Ocupações. Nível de competência: é função da complexidade, amplitude e responsabilidade das atividades desenvolvidas no emprego ou outro tipo de relação de trabalho. Os níveis 1 e 2 compreendem as profissões de competência mais elementares e aquelas sem qualificação.

Segundo a Classificação Internacional Uniforme de Ocupações (CIOU88), 96,3\% exerciam atividades ocupacionais compatíveis com os níveis 1 e 2 de competência, representando as atividades mais elementares e sem qualificação. Os pacientes não exercem atividade profissional, em média, há 12 anos.

No que diz respeito aos diagnósticos, 57,4\% dos pacientes foram incluídos na categoria de esquizofrenia paranoide (CID10). A duração da doença em 76,9\% dos pacientes é superior a 10 anos. Todos fazem uso de medicamentos, média de 4,6 medicamentos diários por paciente. No entanto, 46,3\% relataram sintomas desagradáveis relacionados à sua doença nas 3 semanas precedentes à entrevista (Tabela 2).

Entre as informações sobre a MS dos pacientes, encontrou-se que a média do tempo de internação no hospital de custódia é de 5,5 anos. Os pacientes que estão cumprindo MS por homicídio somam $66,7 \%$. Os outros crimes cometidos incluem: ameaça e tentativa de lesão corporal, atentado violento ao pudor e ato libidinoso, pedofilia, furto, roubo,
Tabela 2. Distribuição dos pacientes nas variáveis clínicas $(n=54)$

\begin{tabular}{|c|c|c|}
\hline Variáveis clínicas & $\mathrm{n}$ & $\%$ \\
\hline \multicolumn{3}{|l|}{ Diagnóstico (CID10) } \\
\hline Esquizofrenia paranoide & 31 & 57,4 \\
\hline Outras* & 23 & 42,6 \\
\hline \multicolumn{3}{|l|}{ Duração da doençä** } \\
\hline$<10$ anos & 12 & 22,2 \\
\hline$\geq 10$ anos & 40 & 74,1 \\
\hline \multicolumn{3}{|c|}{ Internaçōes psiquiátricas anteriores } \\
\hline Sim & 39 & 72,2 \\
\hline Não & 14 & 25,9 \\
\hline \multicolumn{3}{|l|}{ Tempo de internação } \\
\hline$<1$ ano & 18 & 56,3 \\
\hline$\geq 1$ ano & 14 & 43,8 \\
\hline \multicolumn{3}{|l|}{ Uso de medicação psiquiátrica } \\
\hline Sim & 54 & 100,0 \\
\hline Não & 0 & 0,0 \\
\hline \multicolumn{3}{|l|}{ No de medicamentos } \\
\hline$\leq 4$ medicamentos & 27 & 50,0 \\
\hline$\geq 5$ medicamentos & 26 & 48,1 \\
\hline \multicolumn{3}{|l|}{ Remédios trazem algum prejuízo } \\
\hline Sim & 17 & 31,5 \\
\hline Não & 37 & 68,5 \\
\hline \multicolumn{3}{|c|}{ Sintoma desagradável nas últimas 3 semanas } \\
\hline Sim & 25 & 46,3 \\
\hline Não & 29 & 53,7 \\
\hline \multicolumn{3}{|l|}{ Satisfaçäo com a saúde } \\
\hline Nenhuma insatisfação & 36 & 66,7 \\
\hline Alguma insatisfação & 18 & 33,3 \\
\hline \multicolumn{3}{|l|}{ Acompanhamento psicológico } \\
\hline Sim & 40 & 74,1 \\
\hline Não & 13 & 24,1 \\
\hline Não sabe & 1 & 1,9 \\
\hline \multicolumn{3}{|c|}{ Atividade delirante durante a entrevista } \\
\hline Sim & 25 & 46,3 \\
\hline Não & 29 & 53,7 \\
\hline
\end{tabular}

*Estão incluídos: esquizofrenia residual, esquizofrenia hebefrênica, esquizofrenia indiferenciada e esquizofrenia não especificada, de acordo com o CID10; **Tempo médio calculado através do tempo de tratamento do paciente.

incêndio, lesão corporal e tentativa de homicídio (33,3\%). Não existe registro de antecedente criminal em $79,6 \%$ dos casos. Não foram encontradas informações sobre a curatela para $42,6 \%$ dos pacientes. Dentre os pacientes para os quais foi encontrada essa informação, 87,1\% não possuíam curador. E 68,5\% dos pacientes não recebiam visitas. Quanto ao município de origem da sentença, $85,2 \%$ dos pacientes foram identificados como não pertencentes à microrregião na qual está localizado o hospital de custódia.

Respeitando o parecer do Comitê de Ética e Pesquisa da UFMG, foi criado um TCLE para os curadores dos pacientes. Na operacionalização do estudo, deparou-se com a ausência de informação sobre o processo de curatela, bem como da identificação dos supostos curadores. Houve situação em que o curador foi identificado, mas a distância entre os municípios de origem do curador e do hospital de custódia tornou-se um obstáculo para o contato com o mesmo. 
No entanto, a direção da instituição, responsável legal pelos pacientes, foi devidamente esclarecida sobre o estudo, consentindo a sua realização. Nos casos de analfabetismo funcional do paciente, a assinatura do TCLE foi substituída pela impressão digital.

A distribuição dos escores de QV na escala QLS-BR aponta para maior comprometimento no domínio ocupacional. Itens específicos da escala, tais como atividade social, funcionamento ocupacional e nível de realização profissional, tiveram a mediana do escore igual a zero. Dentre os escores apresentados, não houve classificação compatível com uma QV inalterada em nenhum dos itens pesquisados (Tabela 3).

Tabela 3. Distribuição dos escores de qualidade de vida na escala QLS-BR para os pacientes $(n=54)$

\begin{tabular}{lcc}
\hline Domínios, Itens e Escala Global & Mediana & Desvio-padrão \\
\hline Domínio rede social & 1,75 & 1,13 \\
1- Relações familiares & 1,75 & 1,13 \\
2- Relações íntimas & 1,00 & 1,68 \\
3- Amizades ativas & 2,00 & 1,81 \\
4- Atividade social & 0,00 & 0,79 \\
5- Rede social & 1,00 & 1,46 \\
6- Iniciativa social & 2,00 & 1,74 \\
7- Afastamento social & 3,00 & 1,61 \\
Domínio nível ocupacional & 0,25 & 1,66 \\
9- Funcionamento ocupacional & 0,00 & 1,92 \\
10- Nível de realização & 0,00 & 2,00 \\
11- Subemprego & 1,00 & 1,44 \\
12- Satisfação ocupacional & 0,25 & 3,83 \\
17- Utilização do tempo & 0,00 & 1,74 \\
Domínio funçöes intrapsíquicas e relaçöes interpessoais & 2,57 & 0,87 \\
8- Relações afetivo-sexuais & 1,00 & 0,83 \\
13- Sentido de objetivos de vida & 4,00 & 2,25 \\
15- Curiosidade & 1,00 & 1,54 \\
18- Objetos comuns & 2,00 & 0,88 \\
19- Atividades comuns & 1,00 & 0,85 \\
20- Empatia & 4,00 & 1,55 \\
21- Interação na entrevista & 4,00 & 1,45 \\
Qualidade de vida global (21 itens) & 1,74 & 0,89 \\
14- Motivação & 4,00 & 1,89 \\
16- Anedonia & 3,00 & 1,59 \\
\hline
\end{tabular}

Escores $0-1,99=$ QV muito comprometida; Escores $2-4,99=$ QV comprometida; Escores 5 a $6=$ QV inalterada

Na categorização da QV encontrou-se uma concentração dos escores nos itens que indicam comprometimento e excesso de comprometimento da QV dos pacientes (Tabela 4). Nos domínios referentes à rede social e intrapsíquico e de relações interpessoais, não foi encontrado ne- nhum paciente que não apresentasse comprometimento na QV. No domínio ocupacional, foi encontrada a maior porcentagem de pacientes com QV muito comprometida. A escala global, que permite avaliar de maneira geral a QV dos pacientes, mostrou que $61,1 \%$ deles possuíam QV muito comprometida.

Tabela 4. Distribuição dos pacientes de acordo com o escore de qualidade de vida nos domínios da escala QLS-BR em sua forma categorizada $(n=54)$

\begin{tabular}{lcccccc}
\hline $\begin{array}{l}\text { Domínios e Escala } \\
\text { Global }\end{array}$ & $\begin{array}{c}\text { QV muito } \\
\text { comprometida } \\
(0-1,99)\end{array}$ & $\begin{array}{c}\text { QV } \\
\text { comprometida } \\
(2-4,99)\end{array}$ & $\begin{array}{c}\text { QV } \\
\text { inalterada } \\
(5 \mathrm{a} 6)\end{array}$ \\
\hline & $\mathbf{N}$ & $\%$ & $\mathbf{n}$ & $\%$ & $\mathbf{n}$ & $\%$ \\
$\begin{array}{l}\text { Domínio 1* } \\
\text { Rede social }\end{array}$ & 30 & 55,6 & 24 & 44,4 & 0 & 0,0 \\
$\begin{array}{l}\text { Domínio 2** } \\
\text { Nível 0cupacional }\end{array}$ & 39 & 72,2 & 11 & 20,4 & 3 & 5,6 \\
$\begin{array}{l}\text { Domínio 3 } \\
\text { Intrapsíquico e relações interpessoais }\end{array}$ & 15 & 27,8 & 37 & 68,5 & 0 & 0,0 \\
Escala global $\diamond 0$ & 33 & 61,1 & 21 & 38,9 & 0 & 0,0 \\
\hline
\end{tabular}

*Compreende os itens 1 a 7 da Escala QLS-BR; **Compreende os itens 9 a 12 e 17 da Escala QLS-BR; OCompreende os itens 8, 13, 15 e 18 a 21 da Escala QLS-BR.; $\diamond \diamond$ Compreende os itens 1 a 21 da Escala QLS-BR.

Os resultados da análise multivariada estão apresentados por meio da árvore de decisão. Foi definido como variável resposta o escore de QV global na escala QLS-BR. O primeiro galho da árvore mostra o nó raiz com a amostra total de pacientes $(n=54)$. Essa primeira divisão oferece uma estatística descritiva. Observa-se que a média da QV dos pacientes foi de 1,78, valor que corresponde a uma QV muito comprometida (Figura 1).

A variável que melhor discriminou esse grupo foi a duração da doença $(p=0,01)$, mostrando a separação de 2 grupos na árvore de decisão. Para os pacientes com mais de 10 anos de evolução da doença, a média da QV foi de 1,6, e para o grupo com menos de 10 anos de evolução foi de 2,41. Para os pacientes com mais de 10 anos de evolução da doença, o delito cometido mostrou-se muito importante na árvore, apontando que a média da QV daqueles que cometeram homicídio foi de 1,43 e dos que cometeram outros crimes foi de 1,97, ambos com muito comprometimento na QV.

Os pacientes que cometeram homicídio, e que possuem idade inferior a 40 anos, obtiveram a média de 1,74 do escore de QV, a qual, apesar de ser superior à média daqueles com mais de 40 anos $(Q V=1,21)$, também representou $Q V$ muito comprometida.

O ajuste do modelo mostrou que a árvore classificou corretamente $66 \%$ dos entrevistados (Risco $=0,34$ ), resultado que mostra uma boa adequação da análise multivariada para a amostra investigada ${ }^{13}$. 


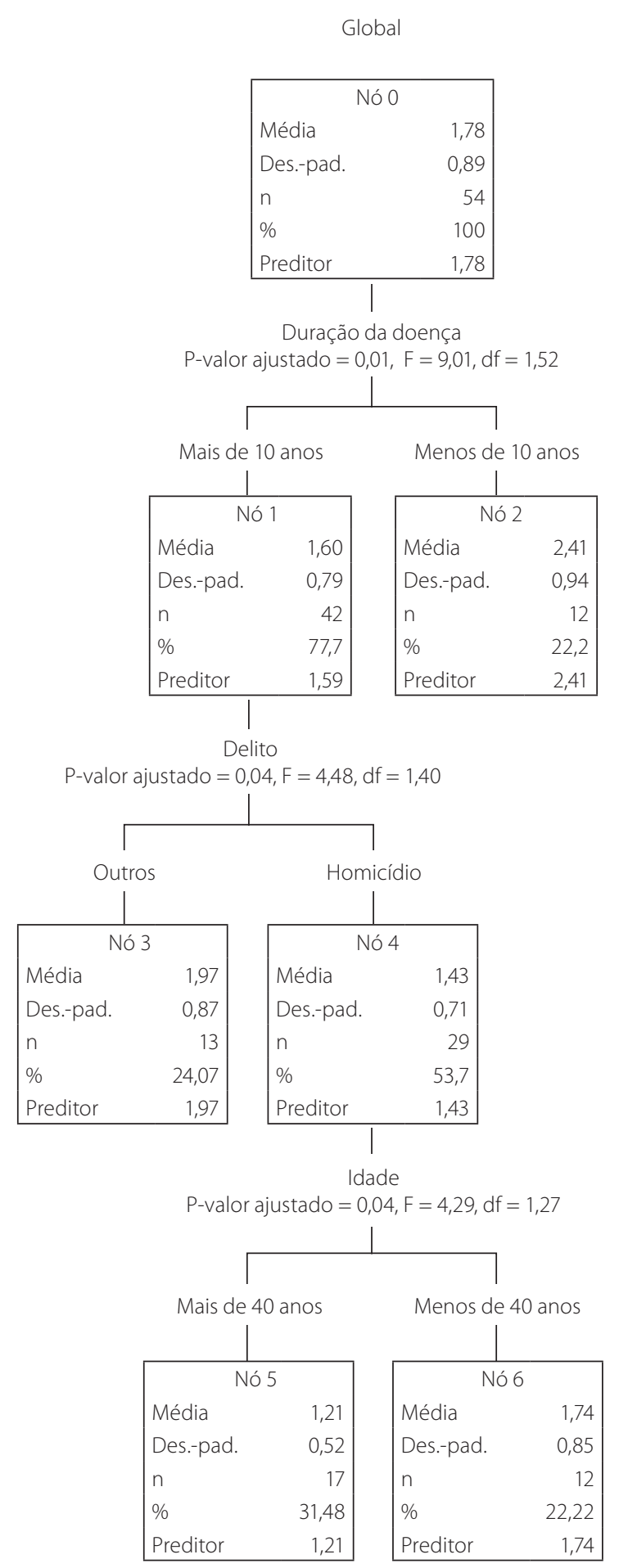

Figura 1. Análise multivariada através da árvore de decisão (CHAID) considerando o escore global de qualidade de vida na escala QLS-BR ( $n=54)$.

Este estudo evidenciou uma baixa QV de pacientes com esquizofrenia cumprindo MS em regime fechado em hospital de custódia. Todos os domínios de QV, inclusive o global, apresentaram escores compatíveis com uma QV muito comprometida, principalmente para o domínio ocupacional.

\section{DISCUSSÃO}

A duração da doença, o tipo de delito cometido e a idade estiveram associados com a QV na análise multivariada. Esses dados apontam para a percepção do impacto causado pela doença e pelo cumprimento da MS em regime fechado na QV dos pacientes.

Na literatura são encontrados estudos que indicam que a esquizofrenia afeta desfavoravelmente a QV de pacientes e familiares. Esses pacientes apresentam uma QV consideravelmente mais baixa, quando comparada com outras populações, principalmente em áreas como trabalho, relacionamentos afetivos, relações familiares e inserção social. A vulnerabilidade às situações estressantes e o baixo comportamento assertivo também são apontados como dificultadores do enfrentamento de situações problemáticas no dia a dia dos pacientes, com impacto na QV 5,12,14,15.

Este estudo evidenciou que a duração da doença interfere significativamente na QV de pacientes com o diagnóstico de esquizofrenia. Outras investigações também apresentam a duração da doença como o principal fator associado à baixa QV nessa população ${ }^{12,16,17}$. Uma maior duração da doença, refletindo em pior $\mathrm{QV}$, revela as consequências da esquizofrenia, somada ao encarceramento decorrente do cumprimento da MS em regime fechado, contribuindo também para os aspectos negativos decorrentes da doença.

De forma coerente com outras investigações, esta pesquisa evidencia pior QV de pacientes com mais idade ${ }^{5,18}$, principalmente no subgrupo com maior duração da doença e que cometeu homicídio.

Embora nesta investigação, a medicação não tenha se mostrado diretamente associada à $\mathrm{QV}$, algumas considerações são importantes. O uso de múltiplos psicofármacos é visto pela literatura como relevante para avaliação da QV, entretanto é difícil estabelecer uma relação direta entre número de medicamentos e uma mais baixa QV, uma vez que indivíduos com diagnóstico de esquizofrenia que usam maior número de medicamentos são aqueles que apresentam quadro mais grave, com maior tempo de evolução da doença, o que reflete em pior QV ${ }^{16}$.

Todos os pacientes deste estudo encontravam-se medicados, usando em média 5 tipos de medicamentos. O relato de reação adversa da medicação foi referido por 31\% dos pacientes. Variável também identificada em outros estudos como associada com prejuízo na QV6,16,19.

Nesta investigação, a maioria dos pacientes $(83,3 \%)$ era solteira. Estudos brasileiros mostram a significativa associação entre o estado conjugal e a QV dos pacientes. A necessidade de um companheiro(a) e constituição de família é apresentada como fator primordial para a QV, sob a ótica dos próprios pacientes ${ }^{6,12,16}$.

Os dados referentes ao recebimento de visitas corroboram com os achados de Fleck et al.9,20, em que 81,2\% dos 584 
pacientes internados em hospital psiquiátrico, que foram pesquisados, não haviam recebido nenhuma visita nos 6 meses anteriores à realização do estudo. Esse achado reflete o processo de isolamento social provocado pelo tempo de internação desses pacientes e, também, a realidade do estigma social, inclusive intrafamiliar, que segrega os portadores de transtorno psiquiátrico à exclusão social.

O resultado de estudos sobre QV de pacientes portadores de transtorno psiquiátrico, incluindo pacientes com diagnóstico de esquizofrenia, mostra melhor QV para pacientes que residem em pensões protegidas se comparados com pacientes hospitalizados. Esses estudos revelam que pacientes submetidos a programas de reabilitação hospitalar relatam significativas melhoras na QV após a saída do hospital ${ }^{2,19}$.

A maioria dos pacientes deste estudo apresentou analfabetismo funcional, denominação dada à pessoa que aprendeu a decodificar minimamente a escrita. Um estudo realizado no Brasil em um hospital psiquiátrico, em que 50\% dos pacientes tinham diagnóstico de esquizofrenia, mostrou que $81 \%$ da população estudada era de analfabetos funcionais ${ }^{18}$. Alguns trabalhos apontam a baixa escolaridade como fator associado ao comprometimento da QV de pacientes com esquizofrenia ${ }^{16,17}$.

A atividade ocupacional é vista na literatura como fator de interferência na QV dos pacientes. A dificuldade encontrada para se inserir no mercado de trabalho formal, as limitações físicas e psicológicas geradas pela doença e, até mesmo, a questão social relacionada à distribuição de tarefas por gênero aparecem como fatores de influência para a baixa QV. A realização de atividades ocupacionais, tais como trabalhos domésticos, trabalhos manuais e trabalhos temporários, é considerada pela literatura como importante preditor para o desenvolvimento de habilidades físicas, autonomia, convívio social e atividades de lazer,12.

Os resultados desta pesquisa apontam para um importante comprometimento do domínio ocupacional. A maioria dos pacientes, antes do início do cumprimento da MS, exercia atividades profissionais de baixa complexidade. O número de pacientes trabalhadores da zona rural e da construção civil evidenciou-se significativo. Tais dados podem estar intimamente relacionados à questão da escolaridade dos pacientes, o que pode ter relação com uma doença insidiosa, com influências negativas nos diversos aspectos da vida dos pacientes.

Os resultados desta investigação trazem importantes contribuições à literatura, no que se refere à QV do paciente com diagnóstico de esquizofrenia em cumprimento de medida de segurança em regime hospitalar. Porém, algumas considerações serão feitas em relação às limitações deste estudo. A primeira delas é a inexistência de outros trabalhos investigando a QV de pacientes em cumprimento de medida de segurança no Brasil, o que impede a comparação dos resultados aqui encontrados. Diante disso, recomenda-se que estudos comparativos sejam conduzidos, de forma a auxiliar na construção de indicadores que subsidiem a escoIha da melhor estratégia terapêutica para essa população, ou seja, se tratamento ambulatorial ou em regime fechado. Instrumentos específicos que contemplem a avaliação do tratamento e da QV de pacientes em cumprimento de MS também precisam ser desenvolvidos ${ }^{21,22}$.

Outra limitação importante é o desenho do estudo transversal, que envolve a questão da temporalidade, o que impossibilita a relação de causalidade. Por exemplo, não se sabe se a baixa QV é decorrente do regime de cumprimento da MS ou se transcende a ele. Outra limitação relacionase ao fato de que nesta investigação avaliou-se apenas a QV de pacientes em cumprimento de MS em regime hospitalar, a qual pode ser diferente para os pacientes em MS com acompanhamento ambulatorial, embora a escala de mensuração de QV utilizada (QLS-BR) ${ }^{10}$, assim como a escala original (QLS) ${ }^{11}$, não apresente advertências em relação a possíveis diferenças de resultados da QV entre pacientes em tratamento ambulatorial e em regime fechado. Portanto, sugere-se a condução de estudos comparativos entre MS em hospital de custódia e regime ambulatorial. Entretanto, há que ser considerado que a qualidade de vida é uma percepção subjetiva do paciente e que as condições estruturais do hospital podem contribuir nessa avaliação.

$\mathrm{O}$ alto percentual de pacientes que apresentam QV muito comprometida, em todos os domínios da escala, inclusive o global, aponta para o chamado efeito chão em instrumentos de avaliação. Esse resultado é reflexo da situação homogênea de falta de exposição dos pacientes aos eventos considerados pertencentes à QV e que fazem parte da escala utilizada. A própria situação de encarceramento em ambiente com características prisionais pode estar interferindo de forma significativa nos valores mínimos da QV dos pacientes avaliados ${ }^{23}$.

\section{CONCLUSÃO}

Diante dos dados apresentados, fica evidente a necessidade de investimento, tanto do meio jurídico como dos serviços de saúde mental, tendo em vista as necessidades dessa população. Estratégias para assistência, promoção e pesquisa sobre QV, de uma maneira geral, precisam ser incentivadas e colocadas em prática nas instituições de saúde mental24. A interlocução entre o sistema prisional e a rede de saúde pública no Brasil tem sido apontada pela literatura como uma importante estratégia para a evolução da saúde coletiva ${ }^{25}$. O serviço prestado nas unidades de tratamento de saúde mental ligadas ao poder judiciário precisa ser avaliado e comparado ao serviço prestado nas unidades civis de atenção, para que possa haver uma maior interação entre as 
propostas de tratamento, bem como para que os princípios e as propostas da reforma psiquiátrica possam atingir e beneficiar os pacientes que cumprem MS.

Novas pesquisas precisam ser conduzidas nas instituições brasileiras para que seja possível uma mudança de paradigma em relação à periculosidade e à inimputabilidade dos portadores de transtorno psiquiátrico. Estudos dessa natureza podem contribuir para a modificação do estigma da periculosidade e para a viabilização de políticas compatíveis com um tratamento mais digno e humanitário para tais pacientes.

\section{REFERÊNCIAS}

1. BRASIL. Código Penal do Brasil. São Paulo: Saraiva; 2001.

2. Goffman E. Manicômios Prisões e Conventos. São Paulo: Perspectiva; 1974.

3. Carrara S. Crime e loucura: 0 aparecimento do manicômio judiciário na passagem do sécuI0. Rio de Janeiro: EDUERJ; 1998.

4. Menezes RS. Psicoses esquizofrênicas. In: Souza CAC, Cardoso RG, editores. Psiquiatria forense: 80 anos de prática institucional. Porto Alegre: Sulina; 2006. p. 213-26.

5. Souza LA, Coutinho ESF. Fatores associados à qualidade de vida de pacientes com esquizofrenia. Rev Bras Psiquiatr. 2006;28(1):50-8.

6. Wagner LC, King M. Existential needs of people with psychotic disorders in Porto Alegre, Brazil. British Journal of Psychiatry. 2005;186:141-5.

7. Cardoso CS, Caiaffa WT, Bandeira M, Siqueira AL, Fonseca IK, Fonseca OPF. Qualidades psicométricas da escala de qualidade de vida para pacientes com esquizofrenia: escala QLS-BR. J Brasil Psiq. 2003;52(3):211-22.

8. Pitta AMF. Qualidade de vida de clientes de serviços de saúde mental. Rev Psiq Clín. 1999;edição especial:68-77.

9. Fleck MP, Wagner L. Avaliação da qualidade de vida na esquizofrenia. In: Fleck MP, editor. Avaliação de qualidade de vida: guia para profissionais de saúde. Porto Alegre: Artmed; 2008. p.147-56
10. Cardoso CS, Bandeira M, Caiaffa WT, Fonseca JOP. Escala de qualidade de vida para pacientes com esquizofrenia (QLS-BR): adaptação transcultural para o Brasil. J Brasil Psiq. 2002;51(1):31-8.

11. Heinrichs DW, Hanlon TE, Carpenter WT. The quality of life scale: an instrument for rating the schizophrenic deficit syndrome. Schizophr Bull. 1984;10:388-98.

12. Cardoso SC, Caiaffa WT, Bandeira M, Siqueira AL, Abreu MNS, Fonseca OPF. Qualidade de vida e dimensão ocupacional na esquizofernia: uma comparação por sexo. Cad Saúde Pública. 2006;22(6):1303-14.

13. Marketing Department SPSS Inc. AnswersTree 3.0. Chicago: User's Guide; 2001.

14. Lehman AF, Ward NC, Linn LS. Chronic mental patients: the quality of life issue. Am J Psychiatry. 1982;139(10):1271-6.

15. Bandeira M, Ireno EM. Reinserção social de psicóticos: avaliação global do grau de assertividade, em situação de fazer e receber crítica. Psicologia: Reflexão e Crítica. 2002;15(3):665-75.

16. Cardoso SC, Caiaffa WT, Bandeira M, Siqueira AL, Abreu MNS, Fonseca OPF. Factors associated with low quality of life in schizophrenia. Cad Saúde Pública. 2005;21(5):1338-48.

17. Coelho VLD, Pantaleão CC. A experiência subjetiva na esquizofrenia: contribuições para uma avaliação sintomatológica. J Bras Psiq. 1999;48(9):393-7.

18. Chan S, Yu lu W. Quality of life of clients with schizophrenia. J Adv Nurs. 2004;45(1):72-83.

19. Campos EM, Caetano D. Qualidade de vida de pacientes esquizofrênicos. J Brasil Psiq. 1998:47:19-22.

20. Fleck MPA, Wagner L, Wagner M. Long-stay patients in a psychiatric hospital in Southen Brazil. Rev Saúde Pública. 2007;41(1):124-30.

21. Schalast N, Redies M, Collins M, Stacey J, Howells K. EssenCES, a short questionnaire for assessing the social climate of forensic psychiatric wards. Crim Behav Ment Health. 2008;18(1):49-58.

22. Nieuwenhuizen Ch Van, Schene AH, Koeter MWJ. Quality of life in forensic psychiatry: an unreclaimed territory. Int Rev Psychiatr. 2002;14(3):198-202.

23. de Soáres PC, Castelo A, Abrão P, Holmes WC, Coconelli RM. Tradução e validação de um questionário de avaliação de qualidade de vida em AIDS no Brasil. Rev Panam Salud Publica. 2009;25(1):69-76.

24. Shiva A, Haden SC, Brooks J. Psychiatric civil and forensic inpatient satisfaction with care: the impact of provider and recipient characteristics. Soc Psychiatry Psychiatr Epidemiol. 2009.

25. Rich JD. Health Issues in prisons and jails: implications for urban health. Journal of Urban Health. 2007:84(3):316-8. 